\title{
COPRIME ARRAY PARAMETERS OPTIMIZATION FOR DOA ESTIMATION
}

\author{
Fatimah A. Salman ${ }^{1}$, Bayan M. Sabbar ${ }^{2}$ \\ 1,2 College of Information Engineering, Al-Nahrain University, Baghdad, Iraq \\ \{fatima.abd, bayan.mahdi\} @ coie-nahrain.edu.iq ${ }^{1,2}$ \\ Received:27/10/2020, Accepted:1/5/2021
}

\begin{abstract}
Sparse array such as the coprime array is one of the most preferable sparse arrays for the direction of arrival estimation due to its properties, like simplicity, the capability of resolving more sources than the number of elements and resistance to mutual coupling issue. In this paper, a new coprime array model is proposed to increase the degree of freedom (DOF) and improve the performance of the coprime array. The newly designed array can avoid mutual coupling by minimizing the lag redundancy and expand the central lags in the virtual difference co-array. Thus, the proposed structure can resolve more sources than the prototype coprime array using the same number of elements with the improved direction of arrival estimation. Simulation results demonstrate that the proposed array model is more efficient than the other coprime array model.
\end{abstract}

keywords: Coprime array, Degree of freedom, Difference co-array, Direction of arrival (DOA) estimation-sparse array

\section{INTRODUCTION}

In array signal processing, an array of elements gathered the received data for estimating the sources signal parameters [1]. The traditional uniform linear array (ULA) consists of $\mathrm{N}$ elements with uniform spacing between the elements that can resolve up to N-1 source or degree of freedom (DOF). To identify more sources, extra elements are added to increase the number of DOF that may lead to increased mutual coupling due to the inter-element spacing which is half the wavelength and complexity [2]. To cope with this situation, different types of the sparse array with the different co-array concept are implemented to resolve up to $O\left(N^{2}\right)$ source using $\mathrm{N}$ elements. As an example, MRA [3], MHA [4], nested array [5], and coprime array [6] are sparse arrays. So as the size of the difference co-array is larger than the number of the physical elements, more uncorrelated sources can be recovered using the DOA estimator. MRA and MHA sparse arrays expanded the size of the difference co-array with a minimum number of holes (empty positions) and reduced the mutual coupling, but they lack the formal expression for the array configuration besides the long processing time to build the physical array [7]. The nested array (NA) and coprime array (CA) focus on these issues and provide large virtual arrays that have a formal expression for the physical array geometry. NA proposed by [5] can generate a hole-free virtual array using two ULAs, one is a dense array with small spacing between the elements and the other is a sparse array. The resulted virtual array suffers from remarkable mutual coupling that declined the DOA estimation performance. One of the most interested sparse array models is the CA that attracts many researchers over the last few. CA consists of two ULA (M,N) with small inter-element spacing between element pairs that eliminate the mutual coupling effect. Lately, different works have been suggested to enhance the CA configuration by maximizing the obtainable DOFs and reducing the mutual coupling affects. In [6], the extended coprime array (ECA) is proposed to get $M N+N-1$ contiguous lags by extending the M-subarray to $2 \mathrm{M}$. The authors in [8] proposed two CA configurations, the former is CACIS in which one sub-array is compressed 
by a compression factor (CF), the latter is CADiS configuration that indicate one subarray is displaced from the other one by a specified distance regarding the $(\mathrm{M}, \mathrm{N})$ pairs value. After reconstructing the array model, different spatial smoothing technique like MUSIC and EEPIRT can be applied to recover the sources which rely on the continuous lags to perceive the signal covariance matrix rank as in [5], [6], [9], [10]. Other works rely on the interpolation technique to manage the holes and exploits of all the unique lags constructed in the virtual array as in [11], [12]. While the CA model is a prospective sparse array model, the main issue is the holes and the inconsecutive ULA lags that directly affects the number of the DOFs [13]. Far from the issue of the holes, different elements pair may produce the same virtual elements in the difference co-array, the number of frequent elements $(\mathrm{m})$ in the difference co-array is called the weight function [14]. So, following some design criteria is an important to construct a sparse array, these criteria include: [9].

- The formal expression that identifies the element positions in an array.

- Large virtual elements in the difference co-array to increase the number of resolvable sources.

- The maximum economic array that means all the elements are essential elements that greatly affect the construction of the difference co-array. MRA, MHA, nested array, and cantor array are examples of maximally economic arrays.

- The weight function had better be small, especially w(1), w(2), and w(3) to alleviate the mutual coupling and root mean square error (RMSE) [9], [15].

In CA with (M,N) elements pair, the number of DOF be enhanced by eliminating the number of lag frequencies in the difference co-array, specifically the cross difference co-array since the self-difference lag frequencies cannot be prevented [16]. In this paper, a new coprime array model is designed based on the above remarks. The proposed array model construct under the coprime array structure accomplishes a higher DOF with reduce mutual coupling and reduced the lag frequencies using the same number of elements as the prototype coprime array.

\section{Signal Model}

For any sensor array with $\mathrm{M}$ elements, the element positioned at $P=(0,1, \ldots M-1) d$, where $\mathrm{d}$ is the spacing between elements and $d=\lambda / 2, \lambda$ is the signal wavelength and the zero position is the reference position. Suppose that $Q$ far-field, uncorrelated narrowband signal is impinging on the array from the direction $\theta_{1}, \theta_{2}, \ldots, \theta_{Q}$, and then the received data at time $t$ is expressed as:

$$
x(t)=\sum_{q=1}^{Q} a\left(\theta_{q}\right) s_{q}(t)+n(t)=A s(t)+n(t)
$$

Where $A=\left[a\left(\theta_{1}\right), a\left(\theta_{2}\right), \ldots a\left(\theta_{q}\right)\right]$ is the array steering vector and, $a\left(\theta_{q}\right)=\left[1, e^{j 2 \pi d \sin \left(\theta_{q}\right)}, \ldots, e^{j 2 \pi(M-1) d \sin \left(\theta_{q}\right)}\right]^{T}, s(t)=$ $\left[s_{1}(t), \ldots, s_{Q(t)}\right]^{T}$ is the source signal vector, and $n(t)$ is the noise vector which is an additive Gaussian white noise with variance $\sigma_{n}^{2}$ that is uniformly distributed. The covariance matrix of the received data $\mathrm{x}(\mathrm{t})$ can be illustrated as:

$$
R=E\left[x(t) x^{H}(t)\right]=A P A^{H}+R_{n}
$$

https://ijict.edu.iq 
Where $P$ is the source signal covariance matrix, $P=E\left[s(t) s^{H}(t)\right]=\operatorname{diag}\left(\left[\sigma_{1}^{2}, \sigma_{2}^{2}, \ldots, \sigma_{Q}^{2}\right]^{T}\right), \sigma_{q}^{2}$ is the signal power of the qth source. $R_{n}$ is the noise covariance matrix, $R_{n}=\sigma_{n}^{2} I_{M}, I_{M}$ is the identity matrix. For an estimated covariance matrix with $T$ snapshots, the sample covariance matrix is expressed as:

$$
\hat{R}=\frac{1}{T} \sum_{t=1}^{T} x(t) x^{H}(t)
$$

\section{A. Difference Co-Array Concept}

For sparse array, to obtain higher DOFs with less number of physical elements, a virtual array is constructed from the difference co-array. Here, some fundamental definition is introduced first.

Definition 1 (Difference Co-array Suppose an array with an integer number of elements positioned at $\mathbb{P}$, the difference set is obtained as follows [5], [17]:

$$
\mathbb{D}=d_{j}-d_{i} \mid d_{j}, d_{i} \in \mathbb{P}
$$

Definition 2(Degree of freedom) For a particular array P, the degree of freedom (DOF) is the number of virtual lags of its difference co-array $(\mathbb{D})$. The uDOF is the uniform DOF that indicates the number of DOF of the central lags of the difference co-array. For a co-array MUSIC, the number of uncorrelated sources that can be resolved is $(u D O F-1) / 2$ [5], [6].

Definition 3 (weight function) For an array $\mathbb{P}$, the weight function $w(m)$ is the number of the elements pair that construct the virtual elements in the difference co-array $\mathbb{D}$ with the index position $(m)$ [18] [9]. It can be described as:

$$
\begin{array}{r}
\mathbb{M}(m)=\left\{\left(d_{j}, d_{i}\right) \in \mathbb{P}\left|d_{j}-d_{i}=m\right| m \in \mathbb{D}\right\} \\
w(m)=\text { length }\{\mathbb{M}(m)\}
\end{array}
$$

The weight function gives an indication of the element allocation in an array. When the weight is larger than one, it refers that there are additional elements pair with a small distance in the physical array that leads to serious mutual coupling [19]. The weight function of the first three elements $w(1), w(2)$ and $w(3)$, which illustrate the number of element pairs can rule the mutual coupling action [20].

By vectoring $\mathrm{R}$ in equation (2), we get

$$
y=\operatorname{vec}(R)=\tilde{A} b+\sigma_{n}^{2} \tilde{i}
$$

Where $\mathrm{y}$ is the virtual measurement of the received vector for the virtual array with central lags for both the positive and negative parts. $\tilde{A}=\left[\tilde{a}\left(\theta_{1}\right), \tilde{a}\left(\theta_{2}\right), \ldots \tilde{a}\left(\theta_{Q}\right)\right], \tilde{a}\left(\theta_{q}\right)=a^{*}\left(\theta_{q}\right) \otimes a\left(\theta_{q}\right)$ is the virtual steering matrix, $\otimes$ represents the Kronecker product, and $\mathrm{b}$ is the signal vector. The corresponding received signal can be found from the difference co-array after discharging the repeated rows. Then, the spatial smoothing technique is applied to the central lags only that have consecutive virtual elements [10]. In this paper, for DOA estimation we use both the spatial smoothing technique and interpolation technique. For spatial smoothing, the Toeplitz matrix is implemented on the contiguous lags to build a full 
rank covariance matrix as follow:

$$
R=\left[\begin{array}{cccc}
(Y)_{\rho} & (Y)_{\rho-1} & . . & (Y)_{1} \\
(Y)_{\rho+1} & (Y)_{\rho} & . . & (Y)_{2} \\
: & : & : & : \\
(Y)_{2 \rho-1} & (Y)_{2 \rho-2} & . . & (Y)_{\rho}
\end{array}\right]
$$

Where $\rho$ refers to the uDOF for the positive side only. $\mathrm{R}$ can be used to estimate the DOA with less multiplication complexity. The nuclear norm minimization interpolation technique mentioned in ref [21] is applied to manage the holes in the difference co-array and exploit all the virtual elements to increase the number of DOF.

\section{B. Prototype Coprime Array Structure}

A prototype coprime array (PCA) is composed of a pair of ULAs (M, N) wavelength as shown in Fig $1 . \mathrm{M}$ and $\mathrm{N}$ are an integer coprime numbers, such that the greater common divisor is one. The distance between the elements in M-subarray is $\mathrm{Nd}$, while the distance between the elements in $\mathrm{N}$-subarray is $\mathrm{Md}$, where $\mathrm{d}$ is the distance which is half the signal [17].

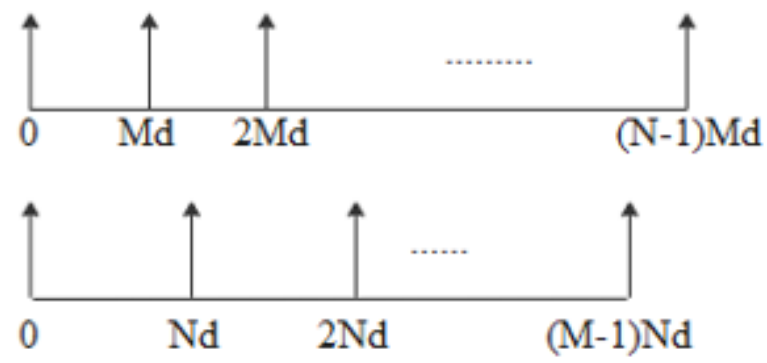

Figure 1: Prototype coprime structure

The physical element's position in the PCA can be expressed in the following sets:

$$
\begin{array}{r}
\mathbb{P}_{1}=\{n M \mid 0 \leq n \leq N-1\} \\
\mathbb{P}_{2}=\{m N \mid 0 \leq m \leq M-1\}
\end{array}
$$

The total number of physical elements is $N+M-1$, since the two subarrays are located at collinear and the first element at zero position is the reference elements for the two subarrays. The difference co-array set of the PCA is given by the self-difference of the two subarrays and the cross difference between the two subarrays as follows:

$$
\begin{array}{r}
\mathbb{D}_{\text {self }}=\left(\mathbb{P}_{1}-\mathbb{P}_{1}\right) \cup\left(\mathbb{P}_{2}-\mathbb{P}_{2}\right), \mathbb{D}_{\text {cross }}=\left(\mathbb{P}_{1}-\mathbb{P}_{2}\right) \cup\left(\mathbb{P}_{2}-\mathbb{P}_{1}\right) \\
\mathbb{D}=\mathbb{D}_{\text {self }} \cup \mathbb{D}_{\text {cross }}
\end{array}
$$

The PCA model has some frequent lags in both the self-difference and the cross-difference. The cross difference lags result from the overlapped elements in $\mathbb{P}_{1}-\mathbb{P}_{2}$ and $\mathbb{P}_{2}-\mathbb{P}_{1}$. The general structure of the difference PCA is demonstrated in Fig. 2. The difference co-array can generate a central lag from $-M-N+1$ to $M+N-1$, several $D O F=M+N-1$ and a lags redundancy $M N-M-N-2[16]$. The first layer hole position in the difference co-array is at $\mathrm{M}+\mathrm{N}$, the 
second layer is at $2 M+N$ and $2 M+N+1$, the third layer at $3 M+N, 3 M+N+1$ and , $3 M+N+2$ [16]. The length of the central lag in the resulted virtual array can't provide the effective number of resolvable DOF after employing the spatial smoothing [22].

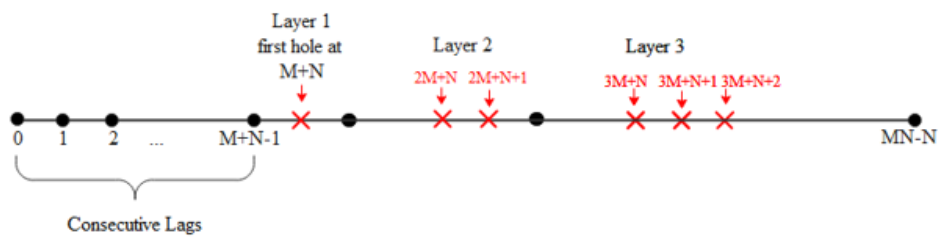

Figure 2: the difference co-array of prototype coprime array

\section{Modified Prototype Coprime ARray Model}

The holes problem and the lag frequencies of the array elements are the main issues considered in designing the proposed array model. The proposed array model does not require additional elements to fill the holes or reduce the lag frequencies. The elements are reallocated in a way that reduces the frequent lags in the difference co-array, fill some holes and enhance the weight unction. The modified model of the PCA structures can be formally expressed for the new array elements position, the contiguous ULA difference co-array, the weight function, and the number of DOFs. From the difference co-array of the PCA, it can be observed that there are common pair's leads to frequent elements in the resulted virtual array. These pairs may lies at the beginning of the two subarrays. We explore this redundancy to construct a new geometry of the PCA as shown in Fig. 3.

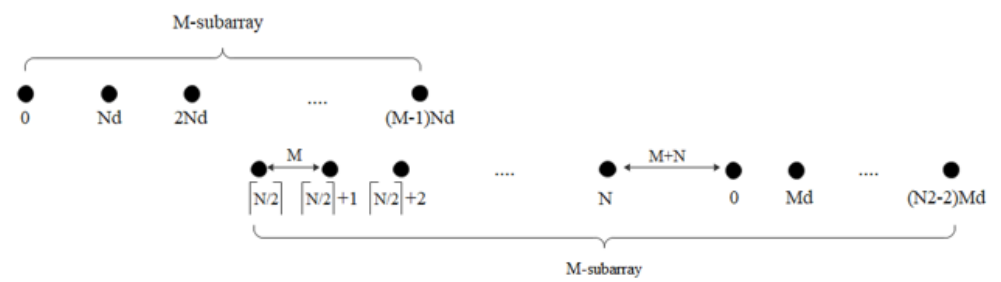

(a)

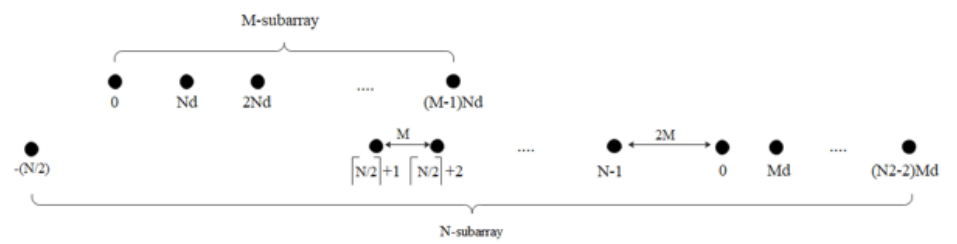

(b)

Figure 3: the proposed array model (a) when $\mathrm{N}$ is the odd value (b) when $\mathrm{N}$ is even value

The proposed PCA model is applied by keeping the M-subarray as in PCA and displacing the first three or more elements of the N-subarray depending on the value of $\mathrm{N}$-subarray whether it's even or odd to new positions since they result in 
overweights function. Besides, they may have less contribution to the central lags of the PCA. After displacing these elements, the position of the new element can be expressed as:

If $\mathrm{N}$ is an odd, then $N 1=\left[\frac{N}{2}\right], N 2=N-N 1$

$$
\begin{array}{r}
\mathbb{P}_{1}=\{m N \mid 0 \leq m \leq M-1\} \\
\mathbb{P}_{2}=\left\{n_{1} M \mid N 1 \leq n_{1} \leq N\right\} \\
\mathbb{P}_{3}=\left\{M N+M+N+n_{2} M \mid 0 \leq n_{2} \leq N 2-2\right\}
\end{array}
$$

If $\mathrm{N}$ is an even, then $N 1=\left[\frac{N}{2}\right], N 2=N-N 1$

$$
\begin{array}{r}
\mathbb{P}_{1}=\{m N \mid 0 \leq m \leq M-1\} \\
\mathbb{P}_{2}=\left\{n_{1} M \mid N 1 \leq n_{1} \leq N-1\right\} \\
\mathbb{P}_{2}=\left\{\frac{-N}{2}\right\} \\
\mathbb{P}_{4}=\left\{M N+M+N+n_{2} M \mid 0 \leq n_{2} \leq N 2-1\right\}
\end{array}
$$

The proposed PCA model has nil cross-difference redundancy and large difference co-array with more unique lags in the resulted virtual array. The number of $\mathrm{UDOF}$ and aperture size of the proposed array model is as listed in Table I.

TABLE I

Number of uDOF and Aperture Size for The Proposed PCA

\begin{tabular}{|c|c|c|}
\hline (M,N)pair & uDOF & Aperture size \\
\hline (M,N-odd) & $M N-M+1$, & $2 M N+N-M-\left[\frac{N}{2}\right] M$ \\
\hline (M,N-even) & $M N+M-N-\left[\frac{N}{2}\right]-1$ & $2 M N-M-(M-1) \frac{N}{2}$ \\
\hline
\end{tabular}

For example, a PCA with $\mathrm{M}=4, \mathrm{~N}=5$, utilize the proposed PCA, the elements are positioned at $[0,5,10,12,15,16,20$, 29] will generate for the positive side, 17 uniform DOF, 22 unique lags, and aperture size is 29 using 8 elements which are more than the traditional PCA, CACIS and the proposed PCA in [16].

\section{Simulation Results}

\begin{tabular}{|c|c|c|c|}
\hline \multicolumn{2}{|l|}{ Array Type } & Aperture Size & $\mathrm{uDOF}$ \\
\hline \multicolumn{2}{|l|}{ PCA } & $M N-M$ & $M+N-1$ \\
\hline \multicolumn{2}{|l|}{ (CACIS), $\mathrm{CF}=2$} & $\mathrm{MN}$ & $M N-(M / C F+1)$ \\
\hline \multicolumn{2}{|l|}{ Reference [16] } & $2 M N-2 N-M+1$ & $2 M+N-1$ \\
\hline \multirow{2}{*}{ Proposed Array Model } & N-odd & $2 M N+N-M-\left[\frac{N}{2}\right] M$ & $M N-M+1$ \\
\hline & N-even & $2 M N-M-(M-1) \frac{N}{2}$ & $M N+M-N-\frac{N}{2}-1$ \\
\hline
\end{tabular}

The performance of the proposed array model is evaluated and compared with PCA, CACIS $(\mathrm{CF}=2)$ and proposed array by [16]. To compare the number of obtainable uDOFs, aperture size and the unique lags using the same number of elements which is $\mathrm{M}+\mathrm{N}-1$, Table II summarize the formal expressions for the proposed array model and the other array configurations

TABLE II

Comparison of Different Array Types Regarding The Aperture Size, uDOF and Unique Lags

A coprime array consists of 8 elements with $\mathrm{M}=4$ and $\mathrm{N}=5$ as shown in Fig. 4. In Fig. 4, the top part shows the position of the physical element, while the bottom part shows the weight function, it illustrates the positive side of virtual difference 
co-arrays for (a) PCA, (b) CACIS, (c) Proposed array by ref[16] and (d) proposed array model. It can be concluded from Fig. 4, that the proposed array model has the cross-difference lags frequency at the virtual element 0 only as of the proposed array model in [16], while the other array configurations have frequent elements lags at different positions.
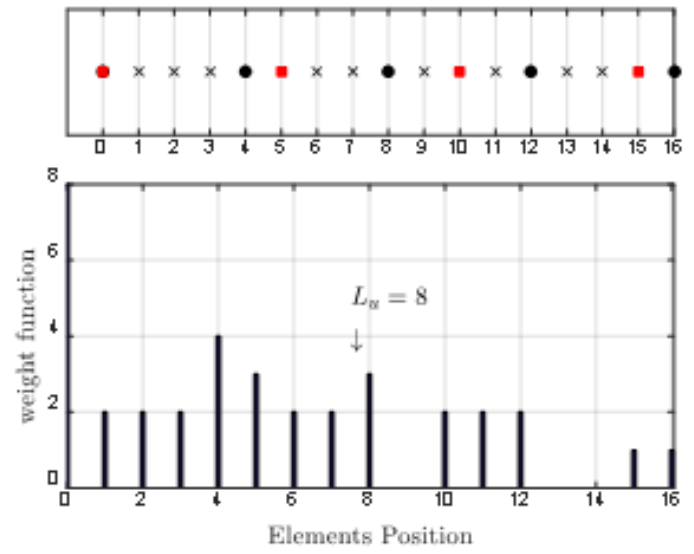

(a)
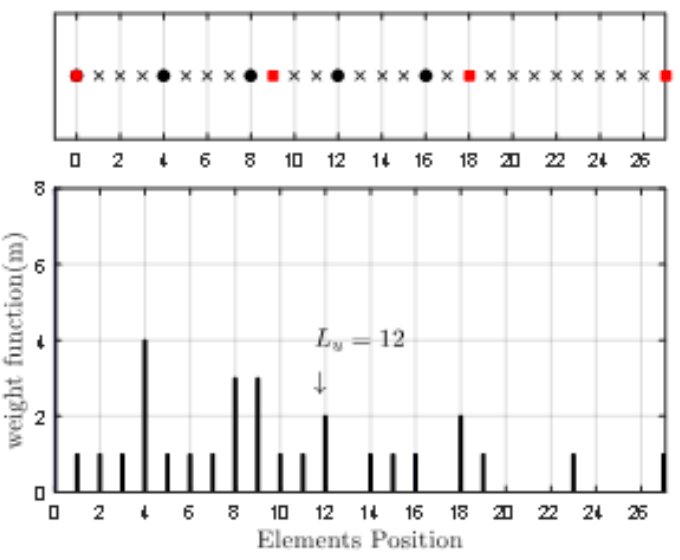

(c)
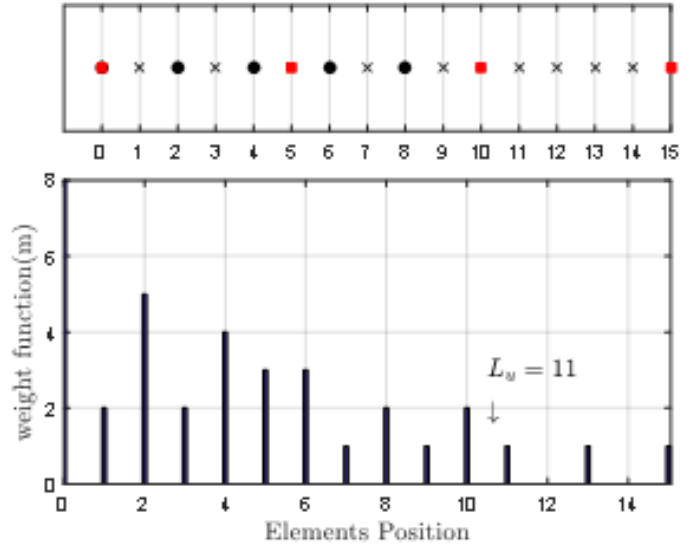

(b)
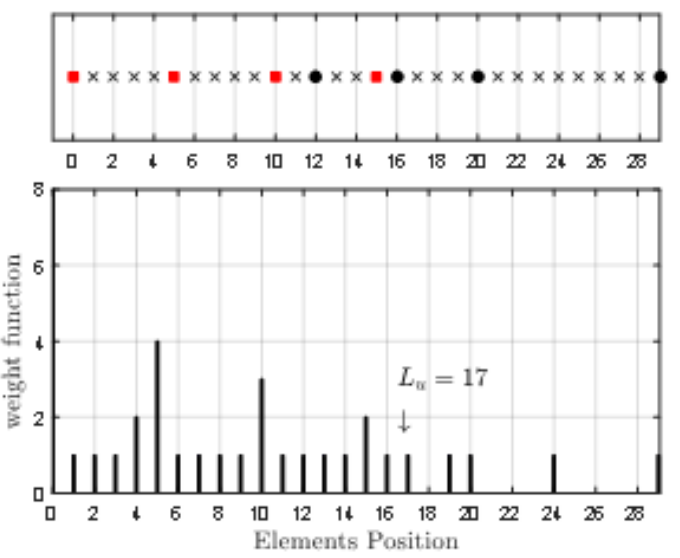

(d)

Figure 4: Elements position and weight function for (a) Prototype coprime array (b) Generalized Coprime array (c) Proposed array [16] (d) Proposed array model, where square red the position of M-subarray element, dot black the N-subarray elements position, $x$ hole

The proposed array model can recover 17 uncorrelated sources successfully since the contiguous is expanded while the PCA, CACIS, proposed array by [16] can recover 8, 11,12 sources respectively using spatial smoothing techniques. The estimation spectrum using spatial smoothing technique for 17 narrowband uncorrelated that uniformly distributed between [-600:600], with SNR=10dB, and the number of snapshots is 500 is demonstrated in Fig. 5. It is clearly shown in Fig. 5 that the proposed array model can detects the entire source successfully with high resolution. Fig. 6 shows the comparison of 
the DOA estimation performance of different array types for 16 uncorrelated sources using the nuclear norm interpolation technique to overcome the problem of the holes in each array using the same number of elements. It can be seen from Fig. 6(a) and (b), that PCA and CACIS can't recover all the sources, there are missing sources. While in Fig. 6(c) and (d), the proposed array in [16] and our proposed array can recover the entire sources successively and with higher resolution. However, the proposed array model can perform better since it presents less RMSE with the generation of higher uDOF and small weight functions that reduce RMSE.

Then, a comparison of the different array configuration is performed regarding the root mean squared error (RMSE). The RMSE is described as :

$$
R M S E=\sqrt{\frac{1}{Q M_{c} \sum_{q=1}^{Q} \sum_{m c=1}^{M_{c}}(\text { estimatedDOA }(m c)-\text { true } D O A)^{2}}}
$$

Where, Mc indicates the number of Monte Carlo trails. Fig. 7 demonstrate the RMSE of different array types for 14 sources that distributed uniformly in the range $(-60 \mathrm{o},-60 \mathrm{o})$ and the number of trials set to 100. Fig. 7(a), shows the RMSE as a function of SNR, where the number of SNR varies from (-20:20) dB with fixed snapshots at 500, while Fig. 7(b) shows the RMSE as a function of different snapshots ranging from (100:1000) with fixed SNR at $10 \mathrm{~dB}$. It can be observed from the figure that the RMSE results of the proposed array model have better performance and a reduction in the RMSE values than other array types. For Fig. 7(a), when the SNR is greater than 5dB, the RMSE is near to zero since it obtains larger contiguous lags of the resulted virtual array. In Fig. 7(b), the RMSE is near zero when the number of snapshots is more than 500 snapshots.

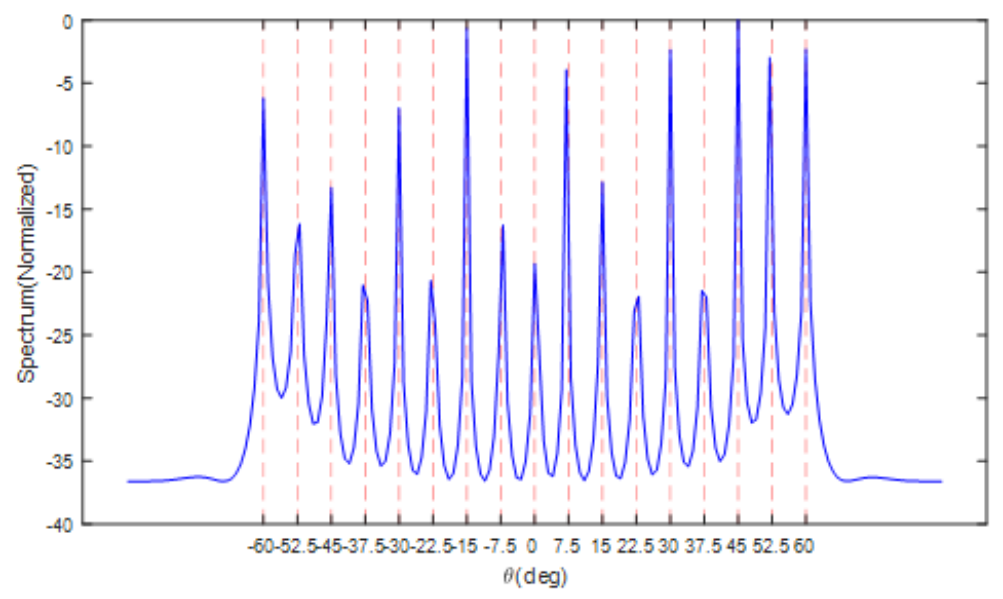

Figure 5: DOA estimation performance of 17 targets with $\mathrm{SNR}=10 \mathrm{~dB}$, snapshot=500 


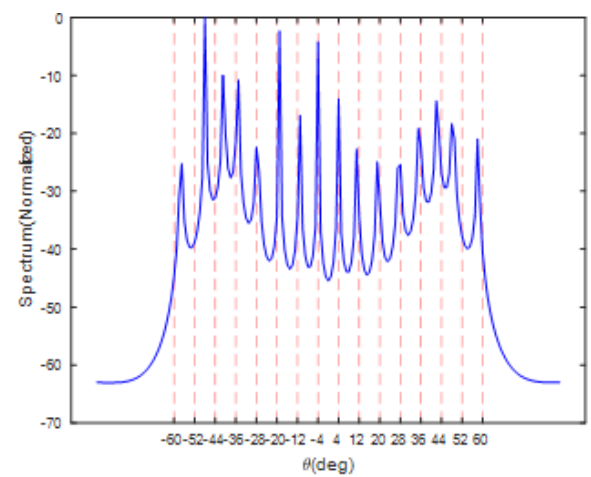

(a) Prototype Coprime Array

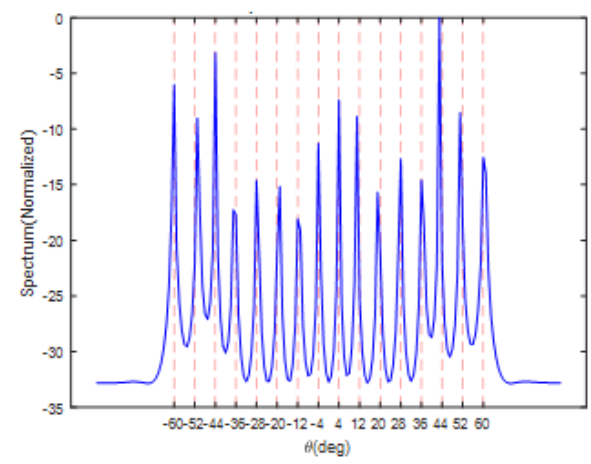

(c) Proposed array by ref[16]

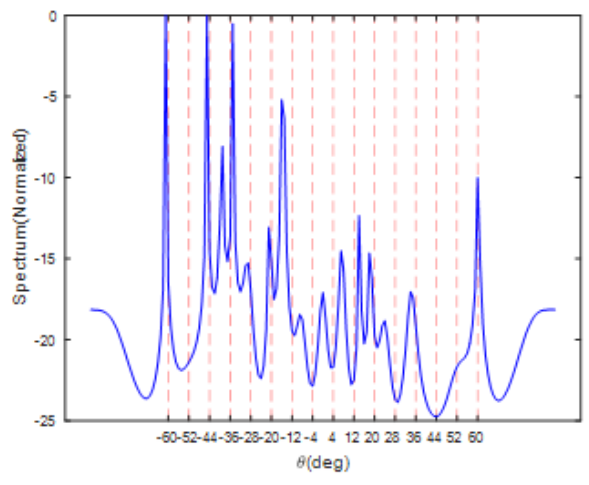

(b) Generalized Coprime Array (CACIS)

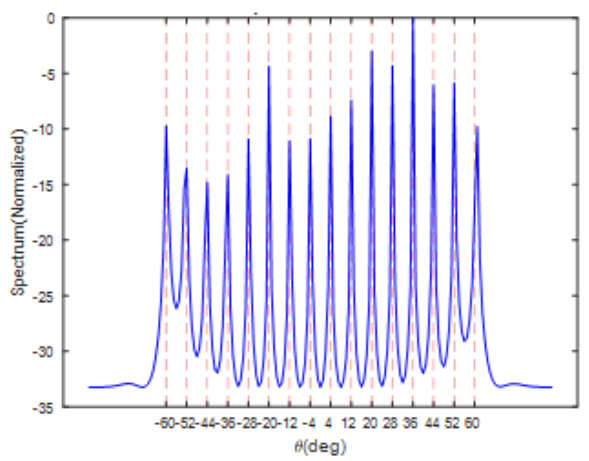

(d) Our Proposed PCA Model

Figure 6: DOA estimation performance of different array configuration using interpolation (nuclear norm)

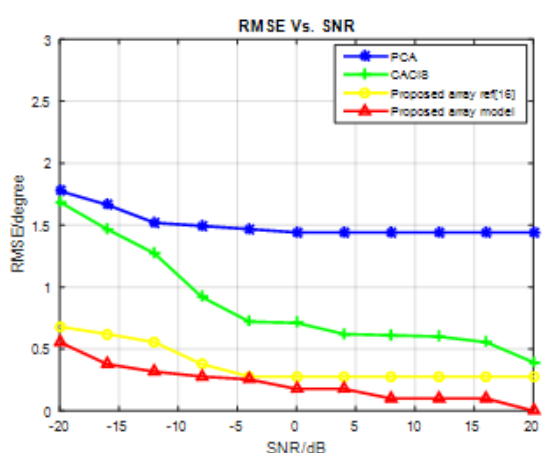

(a) RMSE as a function of SNR for PCA, CACIS, Proposed array ref[16], and our proposed array model, 14 sources, SNAPSHOTS $=500$

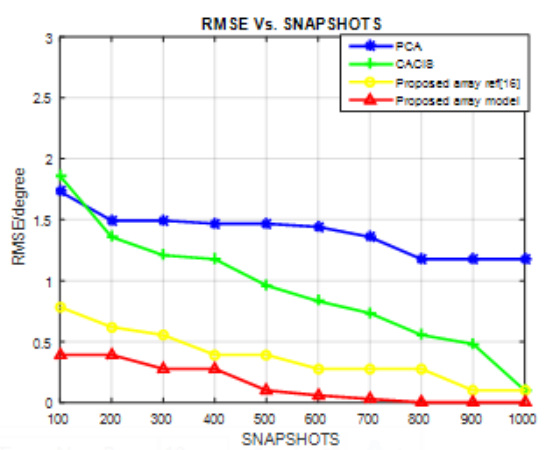

(b) RMSE as a function of SNAPSHOTS for PCA, CACIS, Proposed array ref[16], and our proposed array model, 14 sources, $\mathrm{SNR}=10 \mathrm{~dB}$

Figure 7: The RMSE performance for different array configurations 


\section{Conclusion}

A new coprime array model is proposed by reallocating the position of N-subarray to enhance the number of degree of freedom and improves the DOA estimation. The first three or more elements positions are moved to the end beyond the last element of $\mathrm{N}$-subarray depending on the value of $\mathrm{N}$ (odd or even), the last position in $\mathrm{N}$-subarray and displaced by $\mathrm{M}+\mathrm{N}$ to eliminate the lag frequency and increased the number of uDOF. The resulted array enhances the uDOF using the same number of elements as PCA, CACIS and other arrays. The DOA is performed using the spatial smoothing technique and interpolation technique that reveals better performance and high detection of the resolved signals comparing to other array configurations.

\section{REFERENCES}

[1] Harry L. Van Trees, "Optimum Array Processing: art IV of Detection, Estimation, and Modulation Theory" , 2002.

[2] Z. Shi, C. Zhou, Y. Gu, N. A. Goodman, and F. Qu, "Source Estimation Using Coprime Array: A Sparse Reconstruction Perspective", IEEE Sens. J. , 2017.

[3] T. O. N. Antennas, "Minimum-Redundancy Linear Arrays" , Vol. 2, No. 2, 1968.

[4] G. S. Bloom and S. W. Golomb, "Applications of Numbered Undirected Graphs" , Proc. IEEE, 1977.

[5] P. Pal and P. P. Vaidyanathan, "Nested Arrays: A Novel Approach to Array Processing with Enhanced Degrees of Freedom", IEEE Trans. Signal Process., Vol. 58, No. 8, pp. 4167-4181, 2010.

[6] Piya Pal and P ., "P . Vaidyanathan Dept. of Electrical Engineering" , MC 136-93 California Institute of Technology , Pasadena, CA 91125, USA, Vol. 0, No. 1, pp. 289-294, 2011.

[7] Y. Wang, W. Zheng, X. Zhang, and J. Shen, "Expanded Coprime Array for DOA Estimation: Augmented Consecutive Co-array and Reduced Mutual Coupling", Multidimens. Syst. Signal Process., 2020.

[8] S. Qin, Y. D. Zhang, and M. G. Amin, "Generalized Coprime Array Configurations for Direction-of-Arrival Estimation" , IEEE Trans. Signal Process. , Vol. 63, No. 6, pp. 1377-1390, 2015.

[9] C. L. Liu and P. P. Vaidyanathan, "Super Nested Arrays: Linear Sparse Arrays with Reduced Mutual Coupling-Part I: Fundamentals" , IEEE Trans. Signal Process., Vol. 64, No. 15, pp. 3997-4012, 2016.

[10] C. L. Liu and P. P. Vaidyanathan, "Remarks on The Spatial Smoothing Step in Coarray Music" , IEEE Signal Process. Lett., 2015.

[11] C. Zhou, Y. Gu, X. Fan, Z. Shi, G. Mao, and Y. D. Zhang, "Direction-of-Arrival Estimation for Coprime Array via Virtual Array Interpolation" , IEEE Trans. Signal Process. , 2018.

[12] C. Zhou, Y. Gu, Z. Shi, and Y. D. Zhang, "Off-Grid Direction-of-Arrival Estimation Using Coprime Array Interpolation" , IEEE Signal Process. Lett. , 2018.

[13] Z. Chen, S. Ren, and W. Wang, "DOA Estimation Exploiting Extended Co-Array of Coprime Array" .

[14] Liu, Y. Zhang, Y. Lu, S. Ren, and S. Cao, "Augmented Nested Arrays with Enhanced DOF and Reduced Mutual Coupling", IEEE Trans. Signal Process. , 2017.

[15] C. L. Liu and P. P. Vaidyanathan, "Maximally Economic Sparse Arrays and Cantor Arrays", 2017 IEEE 7th Int. Work. Comput. Adv. Multi-Sensor Adapt. Process. CAMSAP 2017, vol. 2017-Decem, pp. 1-5, 2018.

[16] A. Ahmed, Y. D. Zhang, and J. Zhang, "Coprime Array Design with Minimum Lag Redundancy" , Department of Electrical and Computer Engineering, Temple University, USA Department of Electrical and Computer Engineering , McMaster University , Canada, Vol. 1, pp. 4125-4129, 2019.

[17] P. P. Vaidyanathan and P. Pal, "Sparse Sensing with Co-pprime Samplers and Arrays", IEEE Trans. Signal Process. , Vol. 59, No. 2, pp. 573-586, 2011.

[18] Z. Zheng, W. Q. Wang, Y. Kong, and Y. D. Zhang, "MISC Array: A New Sparse Array Design Achieving Increased Degrees of Freedom and Reduced Mutual Coupling Effect", IEEE Trans. Signal Process., 2019.

[19] X. Wang and X. Wang, "Hole Identification and Filling in K-Times Extended Co-Prime Arrays for Highly Efficient DOA Estimation" , IEEE Trans. Signal Process. , 2019.

[20] I. J. Gupta and A. A. Ksienski, "Effect of Mutual Coupling on the Performance of Adaptive Arrays" , IEEE Trans. Antennas Propag. , 1983.

[21] C. L. Liu, P. P. Vaidyanathan, and P. Pal, "Coprime Coarray Interpolation for DOA Estimation Via Nuclear Norm Minimization" , in Proceedings-IEEE International Symposium on Circuits and Systems, 2016.

[22] W. Du and R. L. Kirlin, "Improved Spatial Smoothing Techniques for DOA Estimation of Coherent Signals" , IEEE Trans. Signal Process. , 1991. 